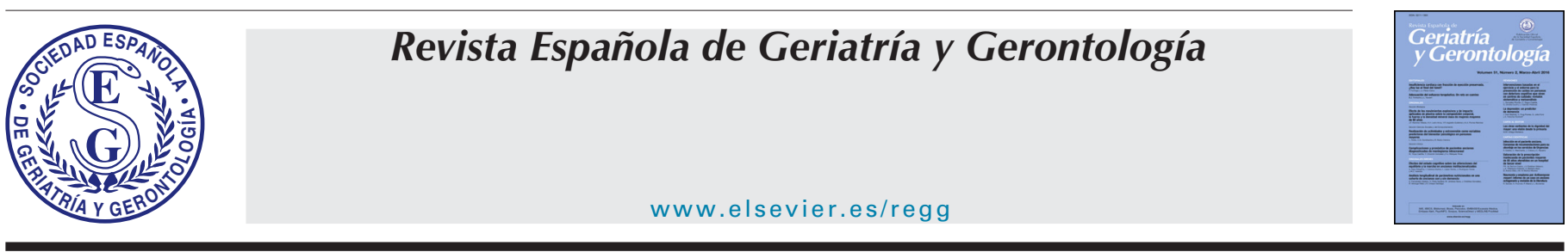

ORIGINAL

\title{
Evidencias psicométricas de una versión breve de la Coping Humor Scale en adultos mayores peruanos
}

\author{
Tomás Caycho-Rodríguez ${ }^{\mathrm{a}, *}$, Mario Reyes-Bossio ${ }^{\mathrm{b}}$, José Ventura-León ${ }^{\mathrm{c}}$, Walter L. Arias Gallegos ${ }^{\mathrm{c}}$, \\ Julio Domínguez-Vergara a y Karla Azabache-Alvarado ${ }^{a}$
}

a Carrera de Psicología, Facultad de Ciencias de la Salud, Universidad Privada del Norte, Lima, Perú

b Facultad de Psicología, Universidad Peruana de Ciencias Aplicadas , Lima, Perú

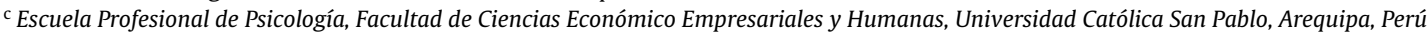

\section{INFORMACIÓN DEL ARTÍCULO}

\section{Historia del artículo:}

Recibido el 3 de febrero de 2018

Aceptado el 9 de septiembre de 2018

On-line el $\mathrm{xxx}$

Palabras clave:

Adultos mayores

Fiabilidad

Humor como afrontamiento

Perú

Validez

\begin{abstract}
R E S U M E N
Introducción: El humor tiene un papel importante en la promoción de un envejecimiento exitoso y saludable. Sin embargo, su estudio científico es aún limitado, en parte debido a la ausencia de medidas validadas para su uso en el ámbito latinoamericano. El objetivo del estudio fue traducir al español y examinar las evidencias de fiabilidad y validez basadas en la estructura interna, convergente y discriminante de la versión peruana de la Coping with Humor Scale-5 ítems.

Materiales y métodos: Los participantes fueron 236 adultos mayores peruanos (78,4\% mujeres y 21,6\% hombres) con un promedio de edad de 72,8 años $(\mathrm{DE}=6,90)$. Se realizó un análisis factorial confirmatorio, análisis de correlación $(r)$ y fiabilidad ( $\alpha$; alfa de cronbach y $\omega$; omega).

Resultados: El modelo unidimensional presentó un ajuste adecuado de los datos $\left(\chi^{2}=7,72, d f=5, p=0,17\right.$, $\chi^{2} / d f=1,54, G F I=0,988, C F I=0,996, N F I=0,988 ; R M S E A=0,048$ [IC90\% 0,000, 0,111]; y SRMR = 0,016). Los índices de fiabilidad son adecuados ( $\alpha=0,86$ [IC95\%: 0,82-0,89] y $\omega=0,89$ [IC95\%: 0,87-0,91]). Se observa una correlación positiva entre la puntuación promedio de la CHS-5 y la satisfacción con la vida, y negativa con la depresión.

Conclusiones: La CHS-5 presenta excelentes propiedades psicométricas, siendo una medida que brinda interpretaciones válidas y confiables para ser utilizada en adultos mayores peruanos.
\end{abstract}

(C) 2018 SEGG. Publicado por Elsevier España, S.L.U. Todos los derechos reservados.

\section{Psychometric evidence of a brief version of the Coping Humor Scale in elderly peruvians}

\begin{abstract}
A B S T R A C T
Introduction: Humour plays an important role in promoting successful and healthy aging. However, its scientific study is still limited, partly due to the absence of validated tools for use in Latin America. The objective of this study was to translate the 5-item Coping with Humor Scale (CHS-5) from English to Spanish and examine the evidence of reliability and validity based on the internal, convergent, and discriminant structure of the Peruvian version of the scale.

Materials and method: The participants were 236 elderly Peruvian adults (78.4\% A confirmatory factor analysis, analysis of correlation (r), and reliability ( $\alpha$, Cronbach's alpha and $\omega$, omega) was performed. Results: The one-dimensional model presented an adequate fit of the data $\left(\chi^{2}=7.72, \mathrm{df}=5, P=.17, \chi^{2}\right.$ / $\mathrm{df}=1.54, \mathrm{GFI}=0.988, \mathrm{CFI}=0.996, \mathrm{NFI}=0.988, \mathrm{RMSEA}=0.048$ [95\% CI; 0.000-0.111]; and SRMR $=0.016$ ). The reliability indexes were adequate $(\alpha=0.86$ [95\% CI: $0.82-0.89$ ] and $\omega=0.89$ [95\% CI: $0.87-0.91])$. A positive correlation was observed between the mean score of CHS-5 and satisfaction with life, and was negative with depression.
\end{abstract}

\footnotetext{
* Autor para correspondencia.

Correo electrónico: tomas.caycho@upn.pe (T. Caycho-Rodríguez).
} 
Conclusions: The CHS-5 has excellent psychometric properties, being a measure that provides valid and reliable interpretations to be used in elderly Peruvians with depression.

(c) 2018 SEGG. Published by Elsevier España, S.L.U. All rights reserved.

Actualmente, se observa un constante crecimiento del número de personas mayores de 60 años, esperando que en el año 2025 lleguen a ser cerca de 1200 millones en todo el mundo ${ }^{1}$. Esta realidad no es ajena a los países latinoamericanos, como Perú, donde se observa un progresivo aumento de la población adulta mayor ${ }^{2}$. Este crecimiento ha generado la formulación de políticas de salud pública a favor de los adultos mayores, fundamentadas en modelos conceptuales de envejecimiento exitoso ${ }^{3,4}$. En relación a esto, el humor ha cobrado un papel importante para la promoción de un envejecimiento exitoso y saludable ${ }^{5,6}$. Simon señala, que para los adultos mayores, el humor tiene un valor terapéutico como mecanismo de afrontamiento ante situaciones estresantes y está relacionado positivamente con la salud percibida y la calidad de vida $^{7}$. Nahemow ${ }^{8}$ reporta que el humor, junto con la tolerancia y la sabiduría, ayudan a brindar un significado positivo al proceso de envejecimiento. Si bien, hasta mediados de la década de 1980, el tema del humor era relativamente ignorado como área de investigación ${ }^{9}$, desde hace algunos años es considerado un factor valioso para el logro de una mejor salud en general ${ }^{10}$.

Definir el humor es complejo debido a sus múltiples concepciones ${ }^{11}$. El humor se puede entender como una reacción emocional positiva ${ }^{12}$ o un rasgo de personalidad ${ }^{13}$. Las diferentes definiciones están en relación con diversos modelos teóricos, entre los que destacan tres principales ${ }^{11}: 1$ ) la teoría de la incongruencia, la cual señala que un conflicto repentino es necesario para la producción de la risa; 2) la teoría de la superioridad, que indica que las personas se ríen de las desgracias de los demás al sentirse superiores; y 3 ) la teoría de la liberación, que afirma que el propósito del humor consiste en aliviar la tensión y los conflictos sociales. Estas tres teorías difieren en el énfasis que le dan a los contenidos del humor y su relación con el contexto social ${ }^{14}$. En este sentido, el modelo de la incongruencia enfatiza la ironía y la sorpresa como contenidos del humor, mientras que el modelo de superioridad se centra en el papel de las relaciones sociales contrarias entre los humoristas y otras personas, grupos u objetos en un contexto determinado.

Actualmente, el humor es comprendido desde una perspectiva multidimensional ${ }^{11}$. Así, Martin ${ }^{15}$ conceptualiza el humor en base a un conjunto de características como 1) la capacidad para comprender chistes y otros estímulos humorísticos, 2) la expresión de humor y alegría, 3) la capacidad de realizar comentarios graciosos, 4) la apreciación de diversos tipos de bromas u otros materiales cómicos, 5) la búsqueda activa de fuentes que provocan risa, 6) el recordar chistes y anécdotas divertidas en la vida y, finalmente, 7) la tendencia a usar el humor como mecanismo de afrontamiento. En relación a esta última característica, estudios recientes ${ }^{16}$ indican que el humor puede actuar como un mecanismo de afrontamiento ante las dificultades aparecidas durante la vejez. Esto proporciona a los adultos mayores la capacidad de cambiar el pensamiento y recuperar una sensación de control, permitiéndoles a su vez adaptarse a los cambios físicos, cognitivos y sociales característicos de esta etapa del ciclo vital ${ }^{17}$.

Un conjunto de investigaciones ${ }^{18-20}$ señala que el humor resulta beneficioso ante la presencia de síntomas de enfermedad física, favoreciendo el sistema circulatorio, respiratorio, muscular, inmunológico y la tolerancia al dolor del adulto mayor. Los beneficios físicos del humor son comparados a los generados por el ejercicio aeróbico ${ }^{21}$. Asimismo, aquellas personas que reconocen con mayor facilidad el humor en su entorno presentan actitudes más positivas frente a la salud física y la enfermedad ${ }^{22}$.

En los adultos mayores, el humor reduce los niveles de ansiedad $^{23}$ siendo beneficioso para el bienestar psicológico ${ }^{18}$, considerándose terapéutico en sí mismo ${ }^{24}$. De igual manera, se ha demostrado que en la vejez es frecuente el efecto del humor como una estrategia de afrontamiento ante el estrés ${ }^{18,21,25-27}$, permitiendo a los adultos mayores distanciarse de las fuentes de estrés mediante la búsqueda de distracciones divertidas y mejorando su salud percibida y calidad de vida ${ }^{18}$. En este sentido, el humor es considerado como un cambio repentino en la perspectiva cognitiva que permite a las personas experimentar alegría y distraerse del estrés que acompaña a una serie de adversidades ${ }^{27,28}$. Los cambios cognitivos a consecuencia del humor permiten a las personas regresar a pensamientos anteriores con una nueva apariencia ${ }^{17}$.

A pesar de la importancia del humor para el afrontamiento del estrés existe una ausencia de medidas validadas en adultos mayores latinoamericanos y específicamente peruanos. Una de estas medidas es la Coping Humor Scale $(\mathrm{CHS})^{28}$, que mide un conjunto de comportamientos directamente relevantes para el uso del humor como una estrategia de afrontamiento. Si bien la CHS fue desarrollada de manera específica para ser aplicada en estudiantes universitarios, se ha empleado también en estudios con adultos mayores ${ }^{18,29,30}$. La CHS conceptualiza el humor como un estilo habitual de utilizar el humor como mecanismo de afrontamiento. Esta definición implicaría que, aunque todas las personas pueden tener la capacidad de usar el humor como estrategia de afrontamiento, pueden variar en su tendencia habitual a utilizarlo.

La versión original de la CHS está en inglés, consta de 7 ítems (CHS-7) y reporta valores del coeficiente al Cronbach que varían entre 0,60 y $0,70^{31}$. Buscando incrementar la consistencia interna, Martin $^{31}$ recomienda dejar de lado el ítem 4 («Tengo que admitir que mi vida sería más fácil si tuviera un poco más sentido del humor»), debido a que las personas parecen interpretarlo de forma inconsistente reduciendo así la fiabilidad. Los resultados de Nezlek y Derks ${ }^{32}$, apoyan la conclusión de Martin ${ }^{31}$ respecto al ítem 4, indicando que el modelo de 6 ítems (CHS-6), excluyendo el ítem 4, es esencialmente unidimensional, presentando además un coeficiente alfa de Cronbach de 0,75. Más recientemente se sugiere eliminar los ítems 1 («A menudo pierdo mi sentido del humor cuando tengo problemas» ) y 4 buscando mejorar la consistencia interna de la versión china de la CHS aplicada a una muestra de ancianos chinos $(\mathrm{CHS}-5)^{30}$. A pesar de esta recomendación, un estudio ${ }^{29}$ utilizando la versión china reportó un valor del coeficiente alfa de Cronbach de 0,60 , similar a la versión original.

En relación a la estructura factorial, el estudio original ${ }^{28}$ no brinda información al respecto, sin embargo, Martín ${ }^{31}$, en base a un análisis de datos de diversas muestras de Canadá, Alemania y los EE. UU., señala que la versión original de la CHS es esencialmente unidimensional. Por otro lado, un estudio ${ }^{33}$ identificó una estructura de dos factores, el primero relacionado con los usos del humor para el afrontamiento y el segundo que expresa el valor que se le da al humor en un contexto de afrontamiento. Los autores sugieren que, a pesar de observarse dos factores el segundo es en esencia una consecuencia del primer factor, por lo que la CHS puede considerarse esencialmente unidimensional y que mide el uso del humor como un mecanismo de afrontamiento. Similares conclusiones han sido reportadas por estudios con versiones cortas de 
5 ítems (CHS-5) $)^{30}$ y 6 ítems (CHS-6) ${ }^{32}$. Todas estas investigaciones concluyen que el humor como afrontamiento, medido por la CHS, es un constructo unidimensional.

A pesar de no existir suficientes estudios antecedentes acerca de las propiedades psicométricas de la CHS-5, resulta valioso contar con un instrumento breve con evidencias de validez y fiabilidad de rápida y fácil aplicación, que generen una buena aceptación y ausencia de fatiga en los adultos mayores evaluados, y cuyos resultados puedan ser rápidamente procesados para su uso con fines de investigación. Asimismo, medidas breves pueden ser incorporadas en evaluaciones masivas por parte de instituciones públicas y privadas que trabajen con adultos mayores permitiendo también evaluar un número mayor de otros constructos, algo difícil si se tienen medidas muy extensas.

Como se mencionó, hasta la fecha son pocos los estudios que informan acerca de las propiedades psicométricas de la CHS-5 en adultos mayores ${ }^{29,30}$. De esta manera, considerando la ausencia de estudios psicométricos de la CHS-5 en el contexto latinoamericano, resulta pertinente plantear la siguiente pregunta de investigación: ¿La CHS-5 mostrará adecuadas propiedades psicométricas en adultos mayores peruanos? En este sentido, el objetivo del presente estudio es analizar la estructura factorial y la fiabilidad de la CHS-5, así como las evidencias empíricas de validez convergente y discriminante a través de la relación con otras variables en una muestra de adultos mayores peruanos. Considerando los argumentos anteriores, las hipótesis fueron las siguientes: 1) los 5 ítems de la CHS-5 conformarán una estructura unidimensional; 2) se observarán correlaciones moderadas entre los 5 ítems de la CHS-5; 3) se presentará una correlación positiva entre las puntuaciones promedio de la CHS-5 y la Escala de Satisfacción con la Vida; 4) se observará una correlación negativa entre las puntuaciones de la CHS-5 y la Escala de Depresión Geriátrica; finalmente, 5) la magnitud de la fiabilidad de la CHS-5 será moderada.

\section{Material y métodos}

\section{Participantes}

Los cuestionarios fueron administrados a 236 adultos mayores asistentes a los Centros del Adulto Mayor (CAM) pertenecientes al Seguro Social de Salud de Perú (EsSalud) de la ciudad de Trujillo. Los participantes fueron seleccionados por un muestreo por conveniencia. Del total de participantes, el 78,4\% fueron mujeres y el $21,6 \%$ hombres. La edad promedio fue 72,8 años ( $D E=6,90)$. El 34,7\% de los participantes estaban casados(as), el 25,8\% eran convivientes, $22,5 \%$ eran viudos(as), el 15,7\% eran divorciados(as)/separados(as), mientras que el 1,3\% eran solteros(as). Asimismo, el 10,6\% vivían solos(as), el 35,2\% vivían solo con su esposo(a), el 26,7\%, vivían con sus hijos, el $25,4 \%$ vivían con su esposo(a) e hijos y finalmente, el 2,1\% vivían con otros familiares. El 48,3\% reportaron tener una buena y muy buena salud física, el $36 \%$ mencionaron poseer una salud física promedio, y finalmente, el 15,7\% reportaron una mala salud física. Respecto a su calidad de vida percibida, el $55,1 \%$ indicaron tener niveles buenos y muy buenos, el 39,4\% manifestaron tener una calidad de vida promedio, mientras que solo el 5,5\% señalaron poseer una calidad de vida mala.

\section{Instrumentos}

Se utilizaron los siguientes instrumentos:

Ficha de datos sociodemográficos. Ficha elaborada por el equipo de investigadores que permite recoger los siguientes datos de los participantes: sexo, edad, estado civil, personas con quien vive, percepción de su salud física y calidad de vida.
Escala de humor como afrontamiento-5 ítems (Coping Humor Scale [CHS-5]). La CHS-5 mide el empleo intencional del humor como estrategia para hacer frente a un conjunto de circunstancias difíciles. En el estudio se utilizó la traducción al español de la versión original de Martin y Lefcourt ${ }^{28}$ con la eliminación de dos ítems, siguiendo lo recomendado por $\mathrm{Ng}$ et $\mathrm{al}^{30}$ para mejorar la consistencia interna. Los cinco ítems presentan cuatro opciones de respuesta que varían de "totalmente en desacuerdo =1" a "muy de acuerdo $=4 »$.

Escala de Satisfacción con la Vida (Satisfaction With Life Scale [SWLS]). Es una medida breve y unidimensional conformada por cinco ítems que mide el juicio global que las personas realizan acerca de la satisfacción con la vida cuyas opciones de respuesta varían de "totalmente en desacuerdo =1" a "totalmente de acuerdo $=5 \%$. Se utilizó la versión española de la $\mathrm{SWLS}^{34}$ de cinco opciones de respuesta tipo Likert, donde una mayor puntuación evidencia una mayor satisfacción con la vida. Para el presente estudio se obtuvo evidencias de fiabilidad adecuada ( $\alpha=0,93$; IC95\%: 0,90-0,94; $\omega=0,93$; IC95\%: 0,92-0,95).

Escala de Depresión Geriátrica-5 ítems (Geriatric Depression Scale [GDS-5]). Medida de cinco ítems ${ }^{35}$ derivada de la escala original de 15 ítems ${ }^{36}$, que permite medir los síntomas depresivos en adultos mayores en las últimas semanas. La GDS-5 presenta opciones de respuesta dicotómicas (SÍ o NO). La fiabilidad para el presente estudio fue adecuada ( $\alpha=0,76$ (IC95\%: 0,70-0,81).

\section{Procedimiento}

El estudio contó con el aval del Comité de ética de la Universidad Privada del Norte, Lima (Perú). En primer lugar, se realizó la traducción al español de la CHS-5 a través de un diseño de traducción inversa buscando asegurar la equivalencia de ambas versiones. Participaron en la traducción dos profesionales bilingües especialistas en psicología que forman parte del equipo de investigación, resolviendo las discrepancias por consenso. Otro miembro del equipo revisó la traducción al español con el objetivo de corregir algunos términos de difícil comprensión para la población adulta mayor. Finalmente, la versión corregida fue aplicada a cinco adultos mayores para realizar las últimas correcciones y conseguir la versión española final (fig. 1).

Los participantes completaron la versión española final de la CHS-5, SWLS y la GDS-5, proporcionando también información acerca de su edad, sexo, convivencia familiar y estado civil. La administración de los instrumentos se realizó a cada anciano de forma individual y en grupos de 5 personas, contando con la autorización de las autoridades de los CAM del Seguro Social de Salud (EsSalud) de la ciudad de Trujillo y teniendo en consideración las normativas éticas y la firma del consentimiento informado de cada uno de los participantes. La tabla 1 permite apreciar la versión original y la versión peruana de la CHS-5.

\section{Análisis de datos}

Se calcularon la media, desviación estándar, asimetría y curtosis para cada uno de los cinco ítems de la CHS-5, así como el coeficiente de Mardia para evaluar la unidimensionalidad multivariada ${ }^{37}$. Considerando lo reportado por la literatura ${ }^{30-32}$, se evaluó un modelo unidimensional de la CHS-5 (un solo factor con los cinco ítems), mediante un análisis factorial confirmatorio (AFC). El AFC se realizó en base a la matriz de correlaciones policóricas y la estimación de máxima verosimilitud robusta, considerando la naturaleza ordinal de los ítems. Se calcularon el estadístico chi cuadrado corregido $\left(\mathrm{SB}-\chi^{2}\right)$, el índice de bondad de ajuste (GFI), índice de ajuste comparativo (CFI), índice de ajuste normalizado (NFI), error cuadrático medio de aproximación (RMSEA) y el residuo cuadrático medio estándar(SRMR) para examinar la bondad de ajuste del modelo de la 


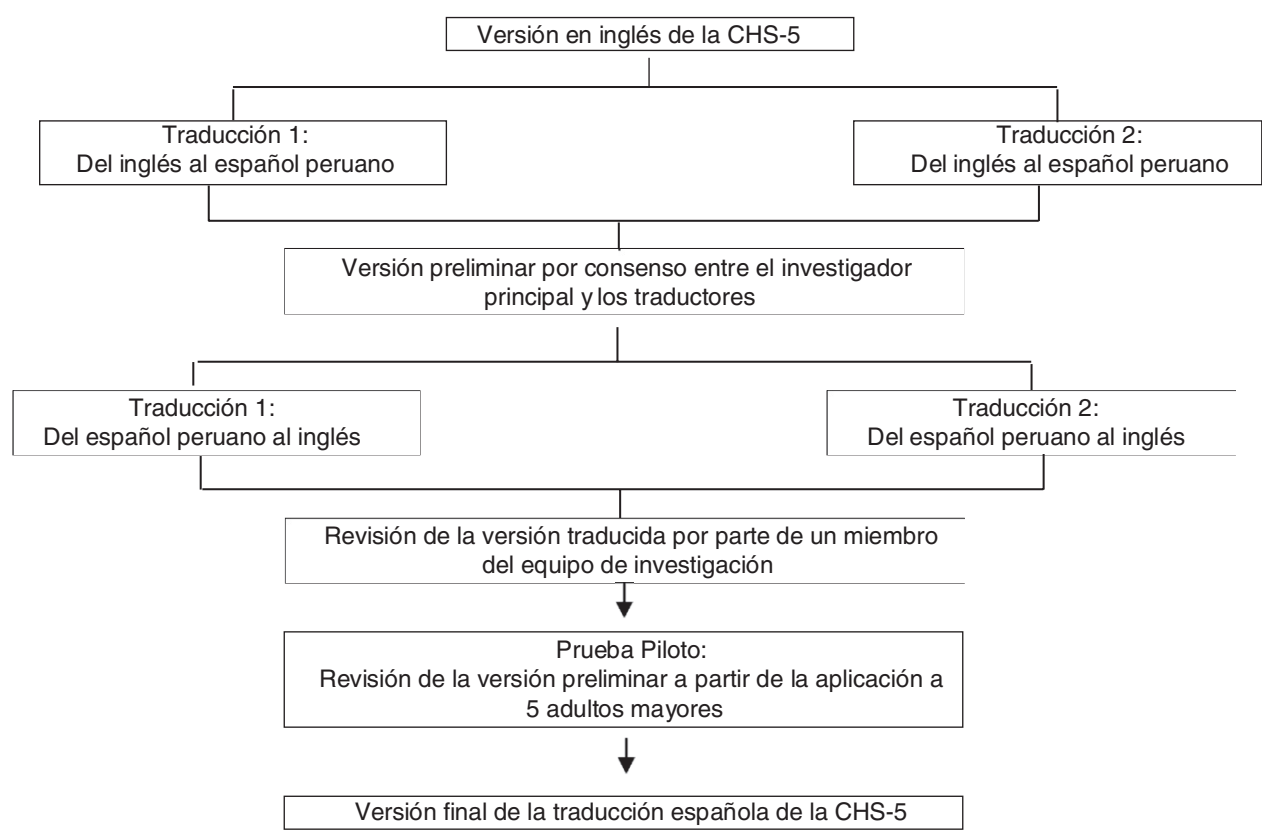

Figura 1. Proceso de traducción de la CHS-5 para su uso en Perú.

Tabla 1

Versión original en inglés y traducción de la versión peruana de la CHS-5

\begin{tabular}{|c|c|}
\hline $\begin{array}{l}\text { Ítems de la versión original en } \\
\text { inglés }\end{array}$ & $\begin{array}{l}\text { Traducción de los ítems en la versión } \\
\text { peruana }\end{array}$ \\
\hline $\begin{array}{l}\text { Ítem 1: } \\
\text { I have often found that my } \\
\text { problems have been greatly } \\
\text { reduced when I tried to find } \\
\text { something funny in them }\end{array}$ & $\begin{array}{l}\text { Ítem 1: } \\
\text { Con frecuencia he encontrado que mis } \\
\text { problemas se reducen en gran parte } \\
\text { cuando he intentado encontrar algo } \\
\text { divertido en ellos }\end{array}$ \\
\hline $\begin{array}{l}\text { Ítem 2: } \\
\text { I usually look for something } \\
\text { comical to say when I am in } \\
\text { tense situations }\end{array}$ & $\begin{array}{l}\text { Ítem 2: } \\
\text { Normalmente busco algo gracioso que } \\
\text { decir cuando me encuentro en } \\
\text { situaciones tensas }\end{array}$ \\
\hline $\begin{array}{l}\text { Ítem 3: } \\
\text { I have often, felt that if I am in a } \\
\text { situation where I have to either } \\
\text { cry or laugh, it's better to laugh }\end{array}$ & $\begin{array}{l}\text { Ítem 3: } \\
\text { Muchas veces he sentido que, en } \\
\text { situaciones que son para reír o llorar, } \\
\text { prefiero reír }\end{array}$ \\
\hline $\begin{array}{l}\text { Ítem 4: } \\
\text { I can usually find something to } \\
\text { laugh or joke about even in } \\
\text { trying situations }\end{array}$ & $\begin{array}{l}\text { Ítem4: } \\
\text { Normalmente encuentro algo de lo que } \\
\text { reírme o hacer chistes, incluso en } \\
\text { situaciones que son para llorar }\end{array}$ \\
\hline $\begin{array}{l}\text { Ítem 5: } \\
\text { It has been my experience that } \\
\text { humor is often a very effective } \\
\text { way of coping with problems }\end{array}$ & $\begin{array}{l}\text { Ítem 5: } \\
\text { En mi experiencia el humor es una } \\
\text { forma muy efectiva de afrontar } \\
\text { problemas }\end{array}$ \\
\hline
\end{tabular}

CHS $-5^{38}$. Considerando que el estadístico $\chi^{2}$ es sensible al tamaño de la muestra ${ }^{39}$, se calculó el estadístico chi cuadrado relativo corregido para el modelo $\left(\mathrm{SB}-\chi^{2} / d f\right)^{40}$, cuyos valores entre 2 y 3 expresan un adecuado ajuste de modelo ${ }^{41}$. Para el CFI, GFI y NFI valores de 0,90 o mayores indican un ajuste aceptable, mientras que valores por encima de 0,95 indican un ajuste excelente. De igual manera, para el RMSEA y SRMR, valores entre 0,05 y 0,08 indican un ajuste aceptable, mientras que valores por debajo de 0,05 brindan mayor evidencia de un excelente ajuste entre el modelo hipotético y los datos empíricos ${ }^{42}$. Respecto a las cargas factoriales estandarizadas $(\lambda)$ valores $\geq 0,50$ son adecuados ${ }^{43}$. Valores de la varianza promedio extraída del factor (Average Variance Extracted) $>0,50$ brindan evidencia interna satisfactoria de validez convergente a nivel del factor $^{44}$
Tabla 2

Medias, desviaciones estándar, asimetría, curtosis y correlación entre ítems de la CHS-5

\begin{tabular}{lllllllll}
\hline Ítem & M & DE & $\mathrm{g} 1$ & $\mathrm{~g} 2$ & \multicolumn{3}{c}{ Correlación interítem } \\
\cline { 5 - 8 } & & & & & Ítem 1 & Ítem 2 & Ítem 3 & Ítem 4 \\
\hline Ítem 1 & 3,16 & 0,87 & $-0,85$ & 0,36 & & & & \\
Ítem 2 & 3,17 & 0,82 & $-0,92$ & 0,49 & 0,61 & & & \\
Ítem 3 & 3,17 & 0,83 & $-0,86$ & 0,23 & 0,63 & 0,72 & & \\
Ítem 4 & 3,27 & 0,77 & $-0,79$ & $-0,00$ & 0,39 & 0,46 & 0,73 & \\
Ítem 5 & 3,13 & 0,69 & $-0,41$ & $-0,07$ & 0,73 & 0,84 & 0,46 & 0,46 \\
\hline
\end{tabular}

DE: desviación estándar; g1: asimetría; g2: curtosis; M: media.

La fiabilidad se calculó utilizando el coeficiente alfa de Cronbach con sus intervalos de confianza al 95\% y el coeficiente Omega $(\omega)^{45,46}$ con sus respectivos IC ${ }^{47}$. Para estimar la evidencia de validez convergente y divergente se calcularon los valores del coeficiente de correlación de Pearson ( $r$ ) entre la puntuación de la CHS-5 y otras medidas que están teóricamente relacionadas con el humor como afrontamiento (satisfacción con la vida y depresión). Se calculó el tamaño del efecto (TE) de las correlaciones, donde $r \geq 0,20$ es TE mínimo recomendado; $\geq 0,50$ TE moderado $y ; \geq 0,80$ TE fuerte, así como IC mediante un módulo MS Excel ${ }^{\circledR}$ ad hoc ${ }^{48}$.

Finalmente, los análisis estadísticos descriptivos y correlacionales fueron realizados con SPSS. 22, mientras que el programa EQS 6.2 fue utilizado para el AFC.

\section{Resultados}

Análisis descriptivo de los ítems de la CHS-5

Las medias, desviaciones estándar, asimetría, curtosis de la CHS-5 se observan en la tabla 2. El ítem cuatro ("Normalmente encuentro algo de lo que reírme o hacer chistes, incluso en situaciones que son para llorar») presenta la mayor puntuación promedio $(M=3,27)$ y el ítem cinco ( $(E n$ mi experiencia el humor es una forma muy efectiva de afrontar problemas») el promedio más bajo $(M=3,13)$. Además, la totalidad de ítems presentan valores de asimetría y curtosis dentro de los límites esperados (entre $\pm 1,5$ ). Se obtiene un valor del coeficiente de Mardia de 5,20 que señala 
el cumplimiento del supuesto de normalidad multivariada ${ }^{49}$, al ser inferior a 35 de acuerdo a la fórmula $p(p+2)$, donde $p$ es el número de variables observadas ${ }^{40}$.

\section{Evidencia de la estructura factorial y estimación} de la fiabilidad de la CHS-5

El AFC realizado en base a las puntuaciones de los cinco ítems de la CHS-5 indicó que el modelo unidimensional presenta índices de ajuste excelentes $\left(\chi^{2}=7,72, d f=5, \mathrm{p}=0,17, \chi^{2} / \mathrm{df}=1,54, \mathrm{GFI}=\right.$ $0,988, \mathrm{CFI}=0,996, \mathrm{NFI}=0,988$; RMSEA $=0,048$ [IC90\% 0,000, 0,111]; y $S R M R=0,016$ ) explicando el $79,9 \%$ de la varianza total. Las cargas factoriales estandarizadas $(\lambda)$ del modelo unidimensional (ítem 1 $=0,73$; ítem $2=0,84$; ítem $3=0,85$; ítem $4=0,85$; ítem $5=0,54$ ), son estadísticamente significativas $(p<0,05)$, en la dirección esperada y con una $\lambda_{\text {promedio }}$ de 0,764 mayor al 0,70 recomendado $^{43}$. Del mismo modo, las correlaciones entre los ítems de la CHS-5 (tabla 1) no son mayores a 0,90 , indicando ausencia de multicolinealidad y brindando evidencias de validez convergente a nivel de factor ${ }^{50}$. Asimismo, un valor de Average Variance Extracted = 0,598 superior a 0,50 permite tener evidencia de validez convergente del factor (es decir, los ítems de la CHS-5 miden un mismo constructo $)^{44}$.

La fiabilidad, estimada con el coeficiente omega ( $\omega=0,89$; IC95\%: $0,87-0,91)$ y alfa de cronbach ( $\alpha=0,86$; IC95\%: 0,82-0,89), brindan evidencia de una fiabilidad adecuada del modelo unidimensional del la CHS-5.

\section{Evidencia de validez convergente y discriminante}

Con el objetivo de examinar evidencias de validez convergente y discriminante se calculó la correlación entre la puntuación de la CHS-5 y las puntuaciones de la SWLS, y GDS-5. Los resultados indican que la puntuación de la CHS-5 correlacionó positivamente con la puntuación promedio de la SWLS $(r=0,826, p<0,01$ [IC95\%: $0,78,0,93])$. Asimismo, se observa una correlación negativa con la puntuación de la GDS-5 ( $r=-0,598, p<0,01$ [IC95\%: -0,51,- 0,81]). Las correlaciones presentan una dirección esperada, son estadísticamente significativas y tienen un TE moderado $(r \geq 0,50)$ y fuerte $(r \geq 0,80)^{48}$.

\section{Discusión}

La medición del humor como afrontamiento en adultos mayores requiere de instrumentos que cuenten con evidencias de validez $\mathrm{y}$ fiabilidad y que, en lo posible, tengan una aplicación breve y sencilla, como es el caso de la CHS-5. En el contexto peruano y latinoamericano no existía ningún instrumento que midiera el humor como afrontamiento en adultos mayores. En este sentido, los resultados demuestran que la CHS-5 cuenta con fiabilidad en sus puntuaciones y evidencia de validez basada en la estructura interna, convergente y discriminante, lo que apoya considerarla como una medida breve del humor como afrontamiento para los adultos mayores en el contexto peruano.

El AFC brinda respaldo empírico que demuestra la unidimensionalidad de la CHS-5, que llega a explicar más del 70\% de la varianza en la muestra total, y la evidencia interna de validez convergente, lo cual está acorde con lo mencionado en otros estudios realizados con adultos mayores de diferentes contextos culturales, pero que no brindaron evidencias objetivas ${ }^{29,30}$. Así, se obtiene una estructura interna adecuada y parsimoniosa de la CHS-5. Asimismo, las correlaciones entre los ítems no son elevadas (ausencia de multicolinealidad entre los ítems), lo que lleva a considerar que los ítems no son redundantes entre sí, favoreciendo la presencia de una solución factorial estable. Por otro lado, si bien el ítem cinco $(\lambda=0,54)$ presenta una carga factorial superior a lo recomendado $(\lambda \geq 0,50)^{42}$ es menor en comparación a los cuatro ítems restantes, indicando que tiene una influencia diferente en la definición del constructo humor como afrontamiento. Esto llevaría a considerar que, para la muestra de adultos mayores peruanos, una adecuada representación de la puntuación de la CHS-5 estaría relacionada con una ponderación diferente de cada ítem ${ }^{51}$.

Respecto a la fiabilidad, esta es adecuada ( $\alpha$ y $\omega>0,80$ ), siendo superior a lo reportado en estudios anteriores con adultos mayores ${ }^{29,30}$. Estos resultados están acordes con lo esperado para la precisión de medidas de tamizaje ${ }^{52}$. En este sentido, para medidas breves como la CHS-5, las evidencias de consistencia interna pueden ser consideradas adecuadas para determinados contextos como los estudios descriptivos, correlacionales o de tipo epidemiológico $^{51}$, pero no para un uso clínico de toma de decisiones, donde se exigen puntuaciones de fiabilidad $>0,90$ a más y donde el error de medida debe ser el mínimo aceptable ${ }^{53}$. En base a lo anterior, la CHS-5 puede ser utilizada en el contexto de la investigación científica, mientras que para mediciones más precisas se hace necesaria una combinación de medidas o instrumentos con mayor cantidad de ítems.

Los resultados de las correlaciones entre las puntuaciones de la CHS-5 y las medidas de SWLS y GDS-5, proporcionan evidencias de validez basada en relación con otras variables tanto en su convergencia y divergencia, al reportar una correlación positiva con la satisfacción con la vida y negativa con la depresión. Estos resultados están en concordancia con estudios que señalan que un mayor uso del humor como estrategia de afrontamiento está asociado con una mayor satisfacción con la vida y menor depresión de los adultos mayores ${ }^{18,54-56}$. Estos resultados podrían estar en relación con ciertas características particulares de los participantes, quienes concurren cotidianamente a los CAM del Seguro Social de Salud de Perú (EsSalud) de la ciudad de Trujillo. En estos contextos sociales, el empleo del humor y la risa permitiría conectar a los participantes entre sí creando un vínculo entre ellos, ser más optimistas y mirar el pasado con menos remordimiento ${ }^{57}$. Así, el humor como estrategia de afrontamiento podría ser utilizado para evitar pensar en las demandas o reevaluar las amenazas en este periodo de la vida ${ }^{58}$. De esta manera, se pone en evidencia el valioso papel del humor en relación con el bienestar de los adultos mayores ${ }^{55,59}$. Resulta importante considerar que una correlación, como la realizada en el presente estudio, no prueba la causalidad entre las variables. Sin embargo, los resultados muestran que el humor como afrontamiento puede ser al menos un indicador de satisfacción con la vida $^{60}$. En este sentido, llevar a cabo programas de intervención para fomentar el empleo del humor como estrategia de afrontamiento pueden ser útiles para mejorar la satisfacción con la vida de los adultos mayores. Esto tendría que ser verificado en estudios posteriores que presenten un diseño adecuado para ello. Los resultados son importantes teniendo en consideración que alrededor del $10 \%$ de los adultos mayores no institucionalizados a nivel internacional presenta algún tipo de trastorno depresivo ${ }^{61}$ que llega a incrementarse a $30,5 \%$ en adultos mayores institucionalizados ${ }^{62}$. En Perú, por ejemplo, se encontró que los adultos mayores institucionalizados tenían menores niveles de felicidad que sus pares no institucionalizados que vivían con sus familias ${ }^{63}$.

En el estudio se observan ciertas limitaciones. Primero, la medición del humor como afrontamiento se realizó de forma transversal, mientras que estudios longitudinales podrían brindar mayor evidencia de validez predictiva. Segundo, los participantes en este estudio fueron adultos mayores no institucionalizados de la ciudad de Trujillo, que, debido a sus características particulares, no representan suficientemente a la totalidad de adultos mayores peruanos. Esto permite evidenciar que próximos estudios centrados en las propiedades psicométricas de la CHS-5 puedan extenderse hacia adultos mayores institucionalizados o con algún tipo de 
enfermedad de diferentes regiones de Perú, con el fin de confirmar la estructura factorial de la CHS-5 en otras muestras de adultos mayores. Cuarto, no se ha analizado las evidencias de invarianza de medida de acuerdo al sexo u otras variables sociodemográficas relevantes debido a la cantidad desigual entre hombres y mujeres. Asimismo, no se ha realizado un análisis de los ítems a través de la teoría de respuesta al ítem que brindaría más información de los mismos. Además, no se estimó la fiabilidad test-retest necesaria para establecer la estabilidad de las puntuaciones de la CHS-5.

A pesar de las limitaciones, la CHS-5 aplicada a adultos mayores peruanos, ha demostrado ser una medida de screnning consistente y adecuada para la medida del humor como afrontamiento, además de ampliar el repertorio de herramientas de medición psicométrica en esta población. Así, este estudio busca servir como inicio para la inclusión del humor como afrontamiento en los estudios acerca de los mecanismos asociados al envejecimiento exitoso y saludable en el contexto peruano y latinoamericano, tal como es reconocido en la literatura internacional ${ }^{5,6,8}$. Finalmente, al contar ya con una medida válida y fiable, futuros estudios deberían ofrecer evidencia, a partir de programas de intervención, del rol del humor como afrontamiento como predictor del envejecimiento exitoso en diferentes contextos.

\section{Conflicto de intereses}

Los autores declaran no tener ningún conflicto de intereses.

\section{Bibliografía}

1. Ward SA, Parikh S, Workman B. Health perspectives: international epidemiology of ageing. Best Pract Res Clin Anaesthesiol. 2011;25:305-17, http://dx.doi.org/10.1016/j.bpa.2011.05.002.

2. Instituto Nacional de Estadística e Informática. Perú: estimaciones y proyecciones de población 1950-2050. Lima: Instituto Nacional de Estadística e Informática; 2016.

3. Rodríguez L. El informe de la Organización Mundial de la Salud sobre envejecimiento y salud: un regalo para la comunidad geriátrica. Rev Esp Geriatr Gerontol. 2016;51:249-51, http://dx.doi.org/10.1016/j.regg.2015.12.013.

4. Cosco TD, Prina AM, Perales J, Stephan BC, Brayne C. Operational definitions of successful aging: a systematic review. Int Psychogeriatr. 2014;26:373-81, http://dx.doi.org/10.1017/S1041610213002287.

5. Chong AML, Ng SH, Woo J, Kwan AYH. Positive ageing: the views of middleaged and older adults in Hong Kong. Ageing \& Society. 2006;26:243-65, http://dx.doi.org/10.1017/S0144686X05004228.

6. Woo J, Ng SH, Chong AML, Kwan AYH, Lai S, Sham A. Contribution of lifestyle to positive ageing in Hong Kong. Ageing Int. 2008;32:269-78, http://dx.doi.org/10.1007/s12126-008-9022-3.

7. Simon JM. The therapeutic value of humor in aging adults. J Gerontol Nurs. 1988;14:8-9, http://dx.doi.org/10.3928/0098-9134-19880801-05.

8. Nahemow L. Humor as a data base for the study of aging. En: Nahemow L, McCluskey-Fawcett KA, McGhee PE, editores. Humor and aging. Orlando: Academic Press; 1986. p. 3-26.

9. Dixon NF. Humor: A cognitive alternative to stress? En: Sarason IG, Spielberger CD, editors. Stress and anxiety, 7. Washington, D.C: Hemisphere; 1980. p. 281-9.

10. Martin RA, Lefcourt HM. Sense of humor and physical health: Theoretical issues, recent findings, and future directions. Humor. 2004;17(1/2):1-20, http://dx.doi.org/10.1515/humr.2004.005.

11. Scheel T. Definitions, theories, and measurement of humor. En: Scheel T, Gockel C, editores. Humor at work in teams, leadership, negotiations, learning and health. New York: Springer International Publishing; 2017, 9-29.

12. Romero EJ, Cruthirds KW. The use of humor in the workplace. Acad Manag Perspect. 2006;20:58-69, http://dx.doi.org/10.5465/AMP. 2006.20591005.

13. Ruch W, Köhler G, van Thriel C. Assessing the humorous temperament: Construction of the facet and standard trait forms of the State-Trait-Cheerfulness-Inventory-STCI. Humor. 1996;9(3-4):303-40, http://dx.doi.org/10.1515/humr.1996.9.3-4.303.

14. FergusonMA, Ford TE. Disparagement humor: A theoretical and empirical review of psychoanalytic, superiority, and social identity theories. Humor. 2008;21:283-312, http://dx.doi.org/10.1515/HUMOR. 2008.014.

15. Martin RA. The psychology of humor: An integrative approach. Burlington, MA: Academic Press; 2007.

16. Schiau I. Humor loneliness and interpersonal communication: A quantitative study of Romanian older adults. Romanian Journal of Communication and Public Relations. 2016;18:89-106.

17. Kruse BG, Prazak M. Humor and older adults: What makes them laugh? J Holist Nurs. 2006;24:188-93, http://dx.doi.org/10.1177/0898010106289855.
18. Celso BG, Ebener DJ, Burkhead EJ. Humor coping, health status, and life satisfaction among older adults residing in assisted living facilities. Aging Ment Health. 2003;7:438-45.

19. Martin RA. Humor, laughter, and physical health: methodological issues and research findings. Psychol Bull. 2001;127:504-19, http://dx.doi.org/10.1037/0033-2909127 4.504.

20. Marziali E, McDonald L, Donahue P. The role of coping humor in the physical and mental health of older adults. Aging Ment Health. 2008;12:713-8, http://dx.doi.org/10.1080/13607860802154374.

21. Berk RA. The active ingredients in humor: Psychophysiological benefits and risks for older adults. Educ Gerontol. 2001;27(3-4):323-39, http://dx.doi.org/10.1080/036012701750195021.

22. Kuiper NA, Nicholl S. Thoughts of feeling better? Sense of humor and physical health. Humor. 2004;17(1/2):37-66, http://dx.doi.org/10.1515/humr.2004.007.

23. Scott CV, Hyer LA, McKenzie LC. The healing power of laughter: The applicability of humor as a psychotherapy technique with depressed and anxious older adults. Soc Work Ment Health. 2015;13:48-60, http://dx.doi.org/10.1080/15332985.2014.972493.

24. Monahan K. The use of humor with older adults aging in place. Soc Work Ment Health. 2015;13:61-9, http://dx.doi.org/10.1080/15332985.2014.918922.

25. Johnson P. The use of humor and its influences on spirituality and coping in breast cancer survivors. Oncol Nurs Forum. 2002;29:691-5, http://dx.doi.org/10.1188/02.0NF.691-695.

26. Kuiper NA, Grimshaw M, Leite C, Kirsh G. Humor is not always the best medicine: Specific components of sense of humor and psychological well-being. Humor. 2004;17(1-2):135-68, http://dx.doi.org/10.1515/humr.2004.002.

27. Wooten P. Humor, laughter and play: Maintaining balance in a serious world En: Dossey B, Keegan B, Guzzetta LC, editores. Holistic nursing: A handbook for practice. Boston: Jones \& Bartlett; 2005. p. 497-520.

28. Martin RA, Lefcourt HM. Sense of humor as a moderator of the relation between stressors and moods. J Pers Soc Psychol. 1983;45:1313-24 http://dx.doi.org/10.1037/0022-3514.45.6.1313.

29. Lai JC, Chong AM, Siu OT, Evans P, Chan CL, Ho RT. Humor attenuates the cortisol awakening response in healthy older men. Biol Psychol. 2010;84:375-80, http://dx.doi.org/10.1016/j.biopsycho.2010.03.012.

30. Ng SH, Woo J, Kwan A, Chong A. Positive ageing: a conceptual framework. Korean J Psychol Soc Issues. 2006;12:29-43.

31. Martin RA. The situational humor response questionnaire (SHRQ) and coping humor scale (CHS): A decade of research findings. Humor. 1996; 9(3-4):251-72

32. Nezlek JB, Derks P. Use of humor as a coping mechanism, psychological adjustment, and social interaction. Humor. 2001;14:395-414, http://dx.doi.org/ 10.1515/humr.2001.011.

33. Thorson JA, Powell FC. Measurement of sense of humor. Psychol Rep. 1991;69:691-702, http://dx.doi.org/10.2466/pr0.1991.69.2.691.

34. Atienza FL, Pons D, Balaguer I, García M. Propiedades psicométricas de la Escala de Satisfacción con la Vida en adolescentes. Psicothema. 2000;12:314-9.

35. Gomez-Angulo C, Campo-Arias A. Geriatric depression scale (GDS-15 and GDS-5): A study of the internal consistency and factor structure. Universitas Psychologica. 2011;10:735-43.

36. Yesavage J, Brink TL. Development and validation of a Geriatric Depression Screening Scale: A preliminary report. J Psychiatr Res. 1983;17:37-49, http://dx.doi.org/10.1016/0022-3956(82)90033-4.

37. Mardia KV. Measures of multivariate skewness and kurtosis with applications. Biometrika. 1970;57:519-30, http://dx.doi.org/10.1093/biomet/57.3.519.

38. Hancock GR, Mueller RD. Structural equation modeling, A second course. Greenwich, Connecticut: IAP; 2006

39. Fujikoshi Y. Transformations with improved chi-squared approximations. J Multivar Anal. 2000;72:249-63, http://dx.doi.org/10.1006/jmva.1999.1854.

40. Bollen KA. Structural equations with latent variables. New York: Wiley; 1989.

41. Carmines EG, McIver JP. Analyzing models with unobservable variables. En: Bohrnstedt G, Borgatta E, editores. Social measurement: Current issues. Beverly Hills, CA: Sage; 1981. p. 65-115.

42. Hu LT, Bentler PM. Cutoff criteria for fit indexes in covariance structure analysis: Conventional criteria versus new alternatives. Struct Equ Modeling. 1999;6:1-55, http://dx.doi.org/10.1080/10705519909540118.

43. Hair J, Anderson R, Tatham R, Black W. Análisis multivariante. México, D.F: Prentice Hall; 2014

44. Fornell C, Larcker DF. Evaluating structural equation models with unobservable variables and measurement error. J Mark Res. 1981;18:39-50, doi: https://10.2307/3151312.

45. McDonald RP. Test theory: A unified treatment. Mahwah, NJ: Erlbaum; 1999.

46. Ventura-León JL, Caycho-Rodríguez T. El coeficiente Omega: un método alternativo para la estimación de la fiabilidad. Revista Latinoamericana de Ciencias Sociales, Niñez y Juventud. 2017;15:625-7.

47. Ventura-León JL. Intervalos de confianza para coeficiente Omega: propuesta para el cálculo. Adicciones. 2018;30:77-8, http://dx.doi.org/ 10.20882/adicciones.962.

48. Caycho-Rodríguez T. Tamaño del efecto e intervalos de confianza para correlaciones: aportes a Montes Hidalgo y Tomás-Sábado. Enferm Clin. 2017;27:331-2, http://dx.doi.org/10.1016/j.enfcli.2017.07.001.

49. Rodríguez MN, Ruiz MÁ. Atenuación de la asimetría y de la curtosis de las puntajes observadas mediante transformaciones de variables: Incidencia sobre la estructura factorial. Psicológica. 2008;29:205-27.

50. Tabachnick BG, Fidell LS, Osterlind SJ. Using multivariate statistics. 4. ta ed. Boston: Allyn \& Bacon; 2001. 
51. Merino-Soto C, Salas E. Escala breve de búsqueda de sensaciones (BSSS): estructura latente de las versiones de 8 y 4 ítems en adolescentes peruanos. Adicciones. 2017. Avance de publicación on-line. 10.20882/adicciones.842.

52. Dominguez-Lara SA, Merino-Soto C. Versión breve de la Escala de afrontamiento ante la ansiedad e incertidumbre preexamen (COPEAU) en universitarios peruanos. Educ Med. 2017, http://dx.doi.org/10.1016/j.edumed.2017.04.011. Avance de publicación on-line.

53. Nunally JC, Bernstein IH. Teoría psicométrica. México, D.F: McGraw-Hill; 1995.

54. Falkenberg I, Buchkremer G, Bartels M, Wild B. Implementation of a manual-based training of humor abilities in patients with depression: A pilot study. Psychiatry Res. 2011;186:454-7, http://dx.doi.org/10.1016/ j.psychres.2010.10.009.

55. Konradt B, Hirsch RD, Jonitz MF, Junglas K. Evaluation of a standardized humor group in a clinical setting: A feasibility study for older patients with depression. Int J Geriatr Psychiatry. 2013;28:850-7, doi: https://10.1002/gps.3893.

56. Shahidi M, Mojtahed A, Modabbernia A, Mojtahed M, Shafiabady A, Delavar A, et al. Laughter yoga versus group exercise program in elderly depressed women: A randomized controlled trial. Int J Geriatr Psychiatry. 2010;26:322-7, doi: https://10.1002/gps.2545.

57. Mathieu SI. Happiness and Humor Group promotes life satisfaction for senior center participants. Act Adapt Aging. 2008;32:134-48, http://dx.doi.org/10.1080/01924780802143089.
58. McCrae RR. Situational determinants of coping responses: loss, threat and challenge. J Pers Soc Psychol. 1984;46:919-28, http://dx.doi.org/10.1037/ 0022-3514.46.4.919.

59. Ruch W, Proyer RT, Weber M. Humor as a character strength among the elderly: Theoretical considerations. Z Gerontol Geriatr. 2010;43:8-12, http://dx.doi.org/10.1007/s00391-009-0080-2.

60. Cappeliez P, O' Rourke N. Empirical validation of a model of reminiscence and health in later life. J Geriatr. 2006;61:237-44, http://dx.doi.org/10.1093/ geronb/61.4.P237.

61. Martínez-de La Iglesia J, Onís-Vilches M, Dueñas-Herrero R, Albert-Colomer C Aguado-Taberné C, Luque-Luque R. Versión española del cuestionario de Yesavage abreviado (GDS) para el despistaje de depresión en mayores de 65 años: adaptación y validación. Medifam. 2002;12:26-40.

62. Conde JL, Jarne A. Trastornos mentales en la vejez. En: Triado C, Villar F (compiladores). Psicología de la vejez. Madrid: Alianza; 2006, p. 341-64.

63. Arias WL, Yépez L, Núñez AL, Oblitas A, Pinedo S, Masías MA, et al. Felicidad, depresión y creencia en la benevolencia humana en adultos mayores institucionalizados y no institucionalizados. Propósitos y Representaciones. 2013;1:83-103. 